\title{
Tumour immunity
}

\section{The lymphoid system and experimental neoplasias}

\author{
A. J. S. DAVIES, B. J. WESTON, R. L. CARTER, AND T. A. CONNORS \\ From the Chester Beatty Research Institute and the Institute of Cancer Research, London
}

In the last decade or so immunologists have left the test tubes in which their serological mysteries were enacted and turned to consider the cells which are involved in immune responses. The resultant cellular immunologists have made discoveries which ought to have lent precision to the quite old notion that the immune mechanism is part of the natural defence system which, in vertebrates, can operate against malignant cells. I intend first to summarize some of the salient findings of the cellular immunologists and secondly to consider a few examples of experimental interaction between the new-look lymphoid system and neoplastic cell populations.

\section{The Lymphoid System}

THE RECIRCULATING LYMPHOCYTE

The lymphocyte not very many years ago was thought of either as a cell of astonishing philoprogenitive versatility or a dull cell with some unspecified part to play in immune responses. In either instance it was commonly supposed that lymphocytes were produced in lymph nodes. This supposition rested on the apparently common sense attitude of, 'where else?'. The experiments of Gowans in the late fifties and early sixties provided the evidence which disposed of some of these misconceptions (Gowans, 1959). Gowans established that at least some lymphocytes were relatively long-lived recirculating cells which could leave the blood stream via postcapillary venules in lymph nodes and return to it via the efferent lymphatics. It seemed that if indeed lymphocytes were produced in lymph nodes it was in relatively small quantities over a long period of time at least in the adult rodent.

\section{THE THYMUS}

In 1961 and 1962, Miller, Good, and Waksman (Miller, 1961; Martinez, Kersey, Papermaster, and Good, 1962a; Papermaster, Dalmasso, Martinez, and Good, 1962a; Waksman, Arnason, and
Jankovic, 1962) all independently showed that the thymus regulated the development of some aspects of immunological responsiveness in young rodents. In mice the ability to reject skin and tumour homografts in particular was grossly impaired by neonatal thymectomy (Miller, 1961; Martinez, Dalmasso, and Good, 1962b). Further, many although not all, humoral immune responses were reduced by the same device; the particular deficit was in relation to the capacity to produce $7 \mathrm{~S}$ antibody (Taylor and Wortis, 1968). In birds homograft rejection was also diminished by early thymectomy but humoral immunity was not affected (Warner and Szenberg, 1962). By contrast, removal of the bursa of Fabricius left homograft reactivity intact but led to failure to respond to antigenic stimulus by production of serum antibodies (Papermaster, Friedman, and Good, 1962b). These findings, taken in conjunction with the studies of the transplantation immunologists, led to consolidation of the concept of two modes of immunity, humoral and cellular, which were not only different functional attributes of the immunological apparatus but differed in their developmental pathways.

\section{THYMUS-DERIVED CELLS}

The early studies on the thymus, whilst giving a broad indication of what the thymus did, failed to illuminate its mechanism of action. There were two mutually compatible hypotheses: first, that the organ secreted a hormone; secondly, that it produced a population of immunologically reactive cells. No formal repudiation of the humoral hypothesis has been possible, and indeed recent evidence (Bach, Dardenne, Papiernik, Barois, Levasseur, and Le Brigand, 1972) suggests that the thymus in both mice and men secretes a factor which can be found in the plasma and which disappears soon after thymectomy. It is too early yet fully to evaluate this new evidence for a humoral factor and it must be stated that more effort has gone into the establishment of the cellular hypothesis. It has been proven, 
in a series of reconstitution experiments using syngeneic but chromosomally marked thymus grafts in mice previously thymectomized, that cells do leave the thymus graft eventually to constitute about half of all lymphocytes in the body (Davies, Leuchars, Wallis and Doenhoff, 1971). These findings are in agreement with results from similar studies based on the distinctive surface antigenicity of thymus-derived cells (Raff, 1971; Raff and Owen, 1971). The other kind of lymphocyte (thymusderived cells are referred to as $T$ cells) has been dubbed a B cell (Roitt, Greaves, Torrigiani, Brostoff, and Playfair, 1969) to indicate (in birds) its bursal origin or (in rodents) that it may derive from a bursal equivalent. The existence of $T$ and $B$ cells has been held further to illustrate that cellular and humoral immunity are separate entities operated by separate cell populations. As I shall emphasize, this concept is of use for purposes of analysis but it is difficult to apply to such complex immune responses as those which can arise in intact animals against tumour homografts.

VARIATION IN THYMUS DEPENDENCY

Most of our thinking about thymus function derives from studies of small rodents. Others have, however, studied rabbits (Sutherland, Archer, and Good, 1964), pigs (Binns, McFarlin, and Sugar, 1972), sheep (Silverstein and Kraner, 1965; Silverstein and Prendergast, 1970; Cole and Morris, 1971a and b), and man (Lischner and DiGeorge, 1969), and it is clear that if any one of these species had been adopted as the type-specimen that our present understanding of the lymphoid system and the immune response would have a very different complexion. Perhaps the most dramatic example is provided by the studies of Cole and Morris and of Silverstein who have emphasized that sheep which have been completely deprived of $T$ cells - during foetal life-can reject skin homografts and can perform nearly all other immunological functions in a normal manner after birth. These experiments can be criticised on the grounds that there are no positive markers for $T$ cells in sheep but if they are interpreted as their authors suggest they give the lie to the dogma that cellular and humoral immunity are two completely different modes of activity operated by separate cell populations one of which is of thymic origin. I think we must accept that our understanding of interspecific and intergeneric variation in immune mechanisms is at present grossly inadequate except to indicate that there is such variation and that extrapolation between species is hazardous. This is particularly true of extrapolation from and to man where clinical information nearly always relates to a pathological state.
COOPERATION IN THE IMMUNE RESPONSE Cooperation was originally used to describe a synergism between $B$ and $T$ cells in relation to the production of humoral antibody. It was thought that B cells produced antibody whereas $T$ cells acted in a helper capacity (Transplantation Reviews, 1969). In those situations where it was possible to distinguish between a carrier and a haptenic component to the antigen it was suggested that $T$ cells responded to the carrier and B cells to the hapten (Raff, 1970). It was envisaged that the $T$ cell bound the carrier to its surface and presented the hapten in some appropriate form to the waiting B cell (Mitchison, Taylor, and Rajewsky, 1970). It is now becoming evident that this early view of cooperation is an oversimplification. Possibly $\mathrm{T}$ cells produce some kind of antibody. Possibly macrophages can bind the ' $T$ ' antibody and thereby trap antigen in such a manner as to present it properly to the $B$ cell (Feldmann, 1972). These notions are too new for proper evaluation but they do show that there is likely to be a complex array of cells which is involved in some kinds of immune response.

\section{Antitumour Immunity}

Good showed in 1962 that neonatally thymectomized mice were more susceptible than their shamthymectomized litter mates to tumour homografts (Martinez et al, 1962b). Miller, Grant, and Roe (1963) found that there was also a slight increase in frequency and in the speed of induction of tumours in neonatally thymectomized mice. This latter point, however, raised the whole issue of immune surveillance and I wish to restrict the discussion to those situations in which there is interaction between an existing tumour and the lymphoid system.

Let me consider two or three simple examples in mice. It is possible to render adult CBA mice grossly deficient in $\mathrm{T}$ cells by a sequence of thymectomy (to ablate the source organ for $\mathrm{T}$ cells), total body irradiation (to destroy existing $\mathrm{T}$ cells), and syngeneic bone-marrow injection (to restore haematopoietic function after irradiation). The resulting radiation chimaeras, sometimes called deprived mice or ' $B$ ' mice to indicate their preponderance of B cells, remain reasonably healthy for over a year. During this time their capacity to make a wide variety of immune responses is grossly impaired but fairly constant.

If two million cells of the EL4 tumour are injected intradermally into such deprived mice the resulting tumour grows rapidly at the site of injection and all the mice die within 20 days. A little over half the animals have metastatic deposits. In the intact control mice the local tumour grows more slowly, 
and metastases are not found. If the tumour cells are injected intravenously into deprived mice death occurs in 14 or 15 days with massive infiltration in viscera, notably the liver, kidneys, and spleen. In normal mice similarly injected, deaths occur late if at all with one or occasionally more tumours, mostly in connective tissues. The EL4 tumour originated in C57B1 mice and it is therefore a homograft in CBA animals. In such circumstances it is clear that the presence of $T$ cells can grossly modify rate and pattern in all allogeneic hosts. If tumours really do differ antigenically from their tissues of origin then it is to be expected that the immune response could affect their growth.

If the Gardner lymphosarcoma which originated in $\mathrm{C} 3 \mathrm{H}$ mice (a strain closely related to CBA) is grown as a solid tumour in deprived CBA animals its growth rate is perhaps a little quicker and it spreads more extensively than in normal CBAs. However, the average life span is similar.

If the R1 or TLX5 tumours, both of which originated in the CBA strain, are transplanted into deprived and normal CBA mice there are no differences in growth rate or pattern of growth of the tumour or survival times of the animals. In these instances it appears that there is little if any effective immunological control over syngeneic tumour growth.

On the basis of these results one might conclude that if there is a big antigenic difference between a tumour and its tissue of origin then the immune response might regulate tumour growth whereas if there is only a small difference, or (heresy) none at all, the immune response is probably irrelevant to tumour growth. The possibility that an immune response to a tumour can actually enhance its growth is here ignored.

However, we must probe a little deeper. It is possible to cure the Gardner lymphosarcoma growing in normal CBA mice by a single intraperitoneal injection of asparaginase (80 iu/ $\mathrm{kg}$ ) 12 days after transplantation of a piece of tumour into the subcutaneous tissues of the flank. The regression that follows is complete and permanent and if this experiment is repeated in deprived mice the tumour regresses in the same way but always reappears within a week and goes on to kill. Further treatment with asparaginase is ineffective and the tumour is asparaginase-resistant when transferred into normal syngeneic hosts (Carter, Connors, Weston, and Davies, 1973). There are many implications of this experiment some of which have been discussed elsewhere (Carter et al, 1973); here I simply want to stress that, although tumour growth was not under effective immunological control, the response to asparaginase was absolutely dependent on the existence of an immune response. The most obvious interpretation is that the chemotherapeutic agent leads to a reduction in the tumour mass and that the immune response, which was unable to cope with the intact tumour, can effectively deal with the new tumour mass. The cured normal mice do not support the growth of subsequent tumour implants, neither has it proved possible to induce a relapse by deprivation after apparent eradication of the tumour.

As far as the RI tumour is concerned, despite its identical growth rate in normal and deprived mice, the response to treatment with methotrexate is better in normal than in deprived animals. Again, the inference is that there is an immunological response against the tumour which is only effective after reduction of tumour volume by chemotherapy. The RI tumour probably arose in a strain of CBA mice which is antigenically distinct from the strain in which we are now using it and it must therefore be thought of as a homograft in the present experiments.

The TLX5 lymphoma arose in the CBA strain in which we are presently growing it. The tumour is sensitive to treatment with bis-dichloroethyl-nitroso urea (BCNU) but this sensitivity is not affected by the immunological state of the host mouse (Connors and Davies, unpublished observations).

These studies show that although growth of transplanted tumours can be affected by the immune response this is by no means a ubiquitous phenomenon. It illustrates that the effectiveness of immunological control can be affected by the tumour mass. Those instances in which chemotherapeutic agents act via the immune response rather than by any direct effect on the tumour are not considered here. Let it suffice that there is an increasing number of such situations.

There now arises the more vexed question of the cellular basis of the putative immunological control over tumour growth. This question is independent of the situations in which no such control is ever exercised. The experiments outlined with deprived mice taken in conjunction with a mass of other evidence illustrate that $T$ cells can somehow control tumour growth. The most obvious way in which they could do this is by developing a cytotoxic capacity which would enable them specifically to kill tumour cells. Such a capability has been amply demonstrated both in vitro and in vivo (Brunner, Nordin, and Cerottini, 1971; Freedman, Cerottini, and Brunner, 1972). There are, however, a number of complications to what would otherwise be a remarkably neat story. First, in some experimental circumstances humoral factors, perhaps antibody or antigen or antigen-antibody complexes, can prevent the killing of target cells by excited T cells. Secondly, it has been shown that target cells which are anti- 
body coated can be killed, and quickly, by B cells (Perlmann and Holm, 1968; MacLennan and Loewi, 1968).

Brief consideration of some of the findings of the Hellströms will now be in place. These investigations have shown that in human patients with a variety of malignant tumours there do exist lymphocytes which are demonstrably cytotoxic in vitro, together with serum factors which can block this cytotoxicity (Hellström and Hellström, 1971). It is widely assumed that the cytotoxic cells are $T$ cells and that the serum factors, if antibody, are B cell products. To complicate the issue it seems likely that the production of blocking antibody may require cooperation between $B$ and $T$ cells for its full expression.

On the basis of this argument antitumour immunity can be seen to be a complex phenomenon with both humoral and cellular components well mixed. It is not inconceivable that there are circumstances in which $\mathrm{T}$ or $\mathrm{B}$ cells predominate and in which rational manipulations of one or other cell population can be achieved. Neither is it beyond the bounds of possibility that some kind of staging on the basis of immunological parameters can be usefully gained. But there are further difficulties which can again be illustrated by an experiment.

If the EL4 (C57B1) tumour is grown as a solid implant in deprived CBA mice which have some time previously been implanted with a chromosomally marked thymus graft it is possible to study the reactive changes in the draining lymph node in such a manner that the mitotic responses of both $B$ and $T$ cells can be followed. This is a situation in which the tumour will grow slowly but progressively and will usually kill the recipient in about 50 days. Mitotic activity of both T and B cells occurs in the first few days after the commencement of tumour growth along with various manifestations of reactive hyperplasia but nearly all this activity dies away by the twentieth day (Weston, Cheers, Carter, Leuchars, Wallis, and Davies, 1972). In the subsequent 30 days or so there are no obvious manifestations of an immunological reaction in the draining nodes except that they are somewhat larger than normal. Current studies indicate that it is possible to induce mitotic responses to other antigens in those nodes which are apparently incapable of continuing to respond to the tumour homograft (Weston, unpublished observations).

The work of the Hellströms indicates clearly that blockage of the effector cells in tumour-bearing organisms is a distinct possiblity. But it seems that in addition some central exhaustion of the capacity to respond to specific transplantation antigens may occur.
In these experiments the difference between the tumour and its host was large in terms of orthodox transplantation antigens. If saturation of the capacity to respond to a slowly growing tumour can be achieved under these circumstances it is likely, though by no means certain, that the specific immunoparesis will be more readily achieved when smaller antigenic differences are involved.

It should be stressed that many of the experimental results presented here relate to more or less rapidly growing long-transplanted tumours. The use of such malignant cell populations for experimental purposes is legitimate but it may be difficult to extrapolate from the results obtained to the human condition in which tumours often grow very slowly and at the time of diagnosis are often much larger in mass than those dealt with in rodents. Much, but not all, of our present knowledge of the immune response against tumours is in fact derived from short-term experiments and acute rather than chronic antigenic stimulation. The latter is less susceptible of analysis than the former and in fact is not fully understood by immunologists who tend to work with less complex antigens than are provided by tumour cells. For these reasons among others few hard and fast conclusions can be drawn at the present time in tumour immunology. The studies presented here illustrate certain principles: (1) Tumour growth rate and metastatic spread can be affected by immune processes. (2) In situations in which chemotherapeutic treatment of a tumour is attempted the immune response which was previously ineffective in controlling tumour growth may be successful in eradicating or controlling the reduced tumour mass. (3) A vigorous immune response may be ineffective because its humoral and cellular components are self-effacing. (4) Immunological exhaustion may arise after which no further augmentation of an antitumour response takes place. This situation is not incompatible with an initially vigorous immune response but simply underlines the notion that whilst tumour growth can be progressive and unrestricted, growth of an antitumour response probably cannot.

These difficulties should not dampen the efforts to characterize tumour-host relationships in immunological terms. It is likely that if we could say in particular instances that there was an active immune response with separately measured humoral and cellular components or that the response was blocked for a particular reason or that there was no evidence of an immunological control that diagnosis, prognosis and treatment would be facilitated.

\section{References}

Bach, J. F., Dardenne, M., Papiernik, M., Barois, A., Levasseur, P., 
and Le Brigand, H. (1972). Evidence for a serum-factor secreted by the human thymus. Lancet, 2, 1056-1058.

Binns, R. M., McFarlin, D. E., and Sugar, J. R. (1972). Lymphoid depletion and immunosuppression after thymectomy in the young pig. Nature [new Biol.], 238, 181-183.

Brunner, K. T., Nordin, A. A., and Cerottini, J. C. (1971). In vitro studies of sensitized lymphocytes and alloantibody-forming cells in mouse allograft immunity. In Cellular Interactions in the Immune Response (2nd International Convocation of Immunology, Buffalo, New York 1970), edited by S. Cohen, G. Cudkowicz, and R. T. McCluskey, pp. 220-230. Karger, Basle.

Carter, R. L., Connors, T. A., Weston, B. J., and Davies, A. J. S. (1973). Treatment of a mouse lymphoma by $\mathrm{L}$-asparaginase. Int. J. Cancer, 11, 345-357.

Cole, G. J., and Morris, B. (1971a). The growth and development of lambs thymectomized in utero. Aust. J. exp. Biol. med Sci., 49, 33-53.

Cole, G. J., and Morris, B. (1971b). The cellular and humoral response to antigens in lambs thymectomized in utero. Aust. J. exp. Biol. med. Sci., 49, 55-73.

Davies, A. J. S., Leuchars, E., Wallis, V., and Doenhoff, M. J. (1971). A system for lymphocytes in the mouse. Proc. roy. Soc. B., 176, 369-384.

Feldmann, M. (1972). Cell interactions in the immune response in vitro. V. Specific collaboration via complexes of antigen and thymus-derived cell immunoglobulin. J. exp. Med., 136, 737760.

Freedman, L. R., Cerottini, J. C., and Brunner, K. T. (1972). In vivo studies of the role of cytotoxic $T$ cells in tumor allograft immunity. J. Immunol., 109, 1371-1378.

Gowans, J. L. (1959). The recirculation of lymphocytes from blood to lymph in the rat. J. Physiol. (Lond.), 146, 54-69.

Hellström, I., and Hellström, K. E. (1971). Cellular immunity and blocking antibodies to tumors. J. Reticuloendothe . Soc., 10, 131-136.

Lischner, H. W., and DiGeorge, A. M. (1969). Role of the thymus in humoral immunity. Lancet, 2, 1044-1049.

MacLennan, I. C. M., and Loewi, G. (1968). Effect of specific antibody to target cells on their specific and non-specific interactions with lymphocytes. Nature (Lond.), 219, 1069. 1070.

Martinez, C., Dalmasso, A. P., and Good, R. A. (1962b). Acceptance of tumour homografts by thymectomized mice. Nature (Lond.), 194, 1289-1290.

Martinez, C., Kersey, J., Papermaster, B. W., and Good, R. A. (1962a). Skin homograft survival in thymectomized mice. Proc. Soc. exp. Biol. (N.Y.), 109, 193-196.

Miller, J. F. A. P. (1961). Immunological function of the thymus. Lancet, 2, 748-749.

Miller, J. F. A. P., Grant, G. A., and Roe, F. J. C. (1963). Effect of thymectomy on the induction of skin tumours by 3,4-benzopyrene. Nature (Lond.), 199, 920-922.

Mitchison, N. A., Taylor, R. B., and Rajewsky, K. (1970). Cooperation of antigenic determinants in the induction of antibodies. In Developmental Aspects of Antibody Formation and Structure, edited by J. Sterzl and I. Riha. Academia, Prague; Academic Press, New York.

Papermaster, B. W., Dalmasso, A. P., Martinez, C., and Good, R. A. (1962a). Suppression of antibody-forming capacity with thymectomy in the mouse. Proc. Soc. exp. Biol. (N.Y.), 111, 41-43.

Papermaster, B. W., Friedman, D. I., and Good, R. A. (1962b). Relationship of the Bursa of Fabricius to immunologic responsiveness and homograft immunity in the chicken. Proc. Soc. exp. Biol. (N.Y.), 110, 62-64.

Perlmann, P., and Holm, G. (1967). Studies on the mechanism of lymphocyte cytotoxicity. In Immunopathology: Mechanisms of Inflammation Induced by Immune Reactions (5th International Symposium on Immunopathology, Punta Ala, 1967), edited by P. Miescher and P. Grabar, p. 325. Schwabe, Basle.

Raff, M. C. (1970). Role of thymus-derived lymphocytes in the secondary humoral immune response in mice. Nature (Lond.), 226, $1257-1258$.

Raff, M. C. (1971). Surface antigenic markers for distinguishing T and B lymphocytes in mice. Transplant Rev., 6, 52-80.

Raff, M. C., and Owen, J. J. T. (1971). Thymus derived lymphocytes their distribution and role in the development of peripheral lymphoid tissues of the mouse. Europ. J. Immunol., 1, 27-30.

Roitt, I. M., Greaves, M. F., Torrigiani, G., Brostoff, J., and Playfair, J. H. L. (1969). The cellular basis of immunological responses; a synthesis of some current views. Lancet, 2, 367-371.

Silverstein, A. M., and Kraner, K. L. (1965). Studies on the autogenesis of the immune response. In Molecular and Cellular Basis of Antibody Formation, edited by J. Sterzl et al, pp. 341-349. Academia, Prague, Academic Press, New York and London.

Silverstein, A. M., and Prendergast, R. A. (1970). Lymphogenesis, immunogenesis and the generation of immunologic diversity. In Developmental Aspects of Antibody Formation and Structure, edited by J. Sterzl and I. Rika. Academia, Prague; Academic Press, New York.

Sutherland, D. E. R., Archer, O. K., and Good, R. A. (1964). Role of the appendix in development of immunologic capacity. Proc. Soc. exp. Biol. (N.Y.), 115, 673-676.

Taylor, R. B., and Wortis, H. H. (1968). Thymus dependence of antibody response: variation with dose of antigen and class of antibody. Nature (Lond.), 220, 927-928.

Transplantation Reviews (1969). Antigen sensitive cells. Transplant. Rev., 1.

Waksman, B. H., Arnason, B. G., and Jankovic, B. D. (1962). Role of the thymus in immune reactions in rats. III. Changes in the lymphoid organs of thymectomized rats. J. exp. Med., 116, 187-206.

Warner, N. L., and Szenberg, A. (1962). Effect of neonatal thymectomy on the immune response in the chicken. Nature (Lond.), 196, 784-785.

Weston, B. J., Cheers, C., Carter, R. L., Leuchars, E., Wallis, V. J., and Davies, A. J. S. (1972). The response of immunologically reconstituted mice to allogeneic skin or tumour grafts. Int. J. Cancer, 9, 66-75. 